\title{
Simulation of a Doppler lidar system for autonomous navigation and hazard avoidance during planetary landing
}

\author{
Scott E. Budge, David B. Chester \\ Center for Advanced Imaging Ladar, Utah State University, \\ Logan, UT 84322-4120, (435) 797-3433
}

\begin{abstract}
The latest mission proposals for exploration of solar system bodies require accurate position and velocity data during the descent phase in order to ensure safe, soft landing at the pre-designated sites. During landing maneuvers, the accuracy of the on-board inertial measurement unit (IMU) may not be reliable due to drift over extended travel times to destinations. NASA has proposed an advanced Doppler lidar system with multiple beams that can be used to accurately determine attitude and position of the landing vehicle during descent, and to detect hazards that might exist in the landing area.

In order to assess the effectiveness of such a Doppler lidar landing system, it is valuable to simulate the system with different beam numbers and configurations. In addition, the effectiveness of the system to detect and map potential landing hazards must be understood. This paper reports the simulated system performance for a proposed multi-beam Doppler lidar using the LadarSIM system simulation software. Details of the simulation methods are given, as well as lidar performance parameters such as range and velocity accuracy, detection and false alarm rates, and examples of the Doppler lidars ability to detect and characterize simulated hazards in the landing site. The simulation includes modulated pulse generation and coherent detection methods, beam footprint simulation, beam scanning, and interaction with terrain.
\end{abstract}

Keywords: Doppler lidar, planetary landing, hazard avoidance, system simulation

\section{INTRODUCTION}

With the advance of solar system body observation missions, one of the important needs is the ability to land safely in a pre-designated site. This task requires an accurate knowledge of position, attitude, and velocity, as well as an understanding of the topology and hazards at the landing site. One of the challenges faced by the navigation system of a lander is drift in the on-board inertial measurement unit (IMU) that inevitably occurs during extended travel times to the destination.

The U.S. National Aeronautics and Space Administration (NASA) has proposed an advanced chirped frequencymodulated continuous-wave (FMCW) Dopplar lidar system to be used during descent to provide estimates of attitude, position, and velocity for safe landing. ${ }^{1-3}$ The system, consisting of an optical head comprising three lidar transmitters that make a $45^{\circ}$ cone angle, enables measurement of both horizontal and vertical velocities, and an estimate of lidar altitude above ground. A prototype of the lidar has been constructed and is under test on the Morpheus free-flyer vehicle.

As the development of the system continues, it is desirable to be able to simulate the performance of the system in various flight paths and with different lidar design parameters. This allows for optimization of performance and to explore various configurations. Another advantage of an accurate simulator is the ability to develop signal processing techniques to exploit the data obtained from the lidar output.

The Center for Advanced Imaging Ladar, at Utah State University, has been developing a lidar simulator for engineering design called LadarSIM ${ }^{4,5}$ since 2003. Originally created to simulate pulsed time-of-flight systems, LadarSIM is capable of simulating the lidar transceiver, focal plane arrays, and pointing/scanning systems, as well as the interaction of the lidar with a simulated 3D scene (including objects). In addition, the software can simulate a moving sensor, allowing for flight trajectories such as aircraft flight, travel on a ground-based platform, and attachment on a descending lander. 
This paper describes the addition of FMCW Dopplar lidar simulation capability to the LadarSIM software. To accomplish the desire to model the NASA planetary body lander concept, LadarSIM has been used to model the multi-beam measurement system during segments of a simulated descent to touchdown. The paper proceeds as follows. Section 2 presents a brief overview of the LadarSIM capabilities. The simulation of the Doppler lidar and multi-beam scanner is presented in Section 3, followed in Section 4 by examples of simulated point clouds created from a model terrain containing both 3D location and measured radial velocity information derived from the lidar simulation. The paper is concluded in Section 5 with a discussion of simulation capabilities.

\section{OVERVIEW OF LADARSIM}

LadarSIM is a lidar system simulation tool created for exploring engineering design tradeoffs during the development of pulsed time-of-flight systems. The main GUI used to run LadarSIM is given in Figure 1. Each of the

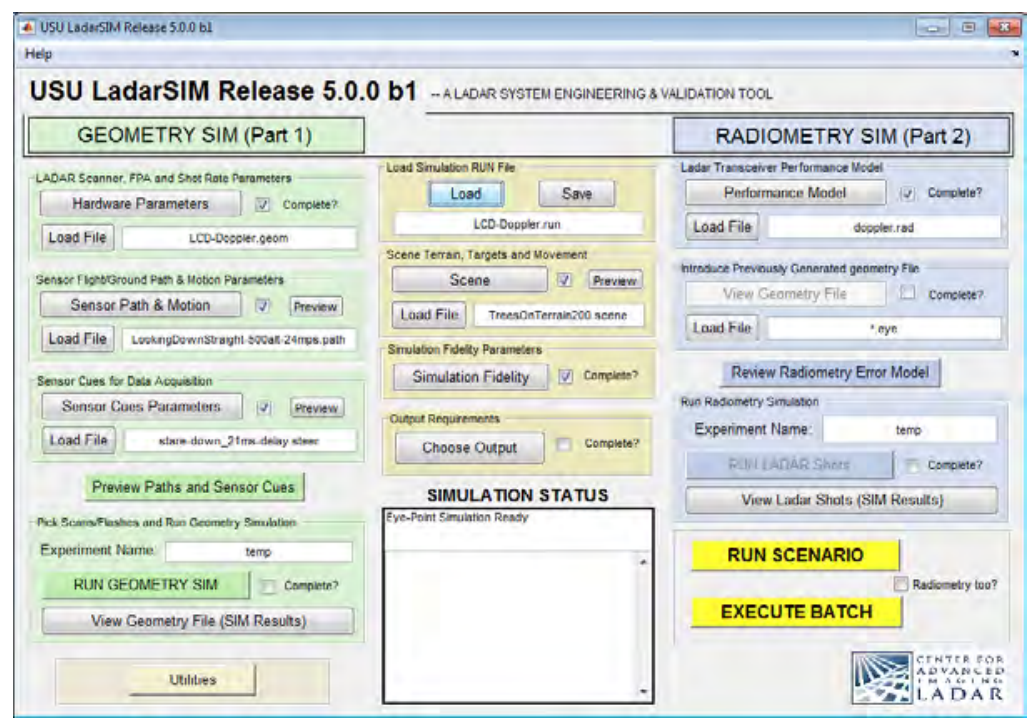

Figure 1: Main GUI for running LadarSIM.

buttons on the GUI are used to open additional GUIs for configuring and running simulations.

In the figure, the center section (colored brown) is used to set basic simulation parameters such as loading in a set of simulation parameters, the level of fidelity of the simulation, and the scene which will be scanned by the lidar. It also includes a Simulation Status window to allow the user to monitor the progress of a simulation.

The left side of the GUI (colored green) allows the user to run a simulation of the lidar system scanner, pointing control, and sensor flight trajectory. The simulation produces a point cloud based solely on the geometric parameters of the scanner and sensor position. This is independent of the type of lidar simulated (e.g. time of flight or FMCW Doppler). With these controls a sensor/flight scenario can be simulated without a transceiver model.

The right side of the GUI (colored blue) can be run after a geometry simulation, and is used to simulate the performance of a specific lidar transceiver configuration, including the signal processing system used to detect 3D points. From this side, simulations of the desired transceiver can be run, which include the type of lidar and lidar system design. By separating the geometry from the radiometry, LadarSIM allows the user to investigate lidar transceiver system tradeoffs without needing to run the scanning and flight scenario more than once.

LadarSIM was modified to provide a FMCW Doppler lidar radiometry simulation, and a multi-beam configuration was modelled in the geometry simulation. 


\section{DOPPLER LIDAR SIMULATION}

\subsection{Proposed Doppler Lidar}

The chirped FMCW lidar simulated in LadarSIM and presented in this paper is based on the NASA lidar used on the Morpheus test-bed. ${ }^{1}$ There are many sources for a basic understanding of chirped FMCW radar/lidar (e.g. Booker ${ }^{6}$ ). The lidar makes use of a frequency-chirped modulated laser, in a homodyne configuration with a balanced detector. The advantages of this system design are described by Adany. ${ }^{7}$

The transmitted laser waveform consists of an up-chirp from frequency $F_{1}$ to frequency $F_{2}$, followed by a constant frequency segment to collect Doppler information, followed by a down-chirp from $F_{2}$ to $F_{1}$. These three segments can then be processed to determine the Doppler frequency, which gives velocity information, and the beat frequencies from which range is derived. Both an up-chirp and a down-chirp are needed to resolve the ambiguity in the radial speed of the target with respect to the lidar (either moving towards the target, or moving away from the target).

The chirped signal is transmitted to the target, and the delayed return signal is optically mixed in the detector to produce a beat frequency spectrum, $f_{b}$, which is proportional to the range to the target. For a target moving towards the lidar, and when Doppler frequency $f_{d}$ is less than $f_{b}$, the frequency seen after the mixer during the up-chirp is given by $f^{+}=f_{b}-f_{d}$, and the frequency seen after the mixer during the down-chirp is given by $f^{-}=f_{b}+f_{d}$. The range and radial speed of the target are given by

$$
R=\left(\frac{T c}{2 B}\right)\left(\frac{f^{+}-f^{-}}{2}\right) \quad v_{r}=\left(\frac{\lambda}{2}\right)\left(\frac{f^{+}+f^{-}}{2}\right)
$$

where $B$ is the modulation bandwidth, $T$ is the chirp waveform period, $c$ is the speed of light, and $\lambda$ is the laser wavelength. The ambiguity arises when the sign of $v_{r}$ is unknown or when $f_{d}>f_{b}$.

The NASA lidar design has three beams forming a cone angle of $45^{\circ}$. Since each beam is offset from nadir, it is possible to combine the radial velocities measured from each beam to estimate a velocity vector for the platform, and to combine the ranges to estimate the altitude and orientation of the platform above the surface.

\subsection{Scanner Simulation}

There is interest in studying the advantages of different numbers of beams and different beam configurations. The scanner simulated for this paper is a non-mechanical scanner being developed by Fibertek, Inc. The initial design increases the number of beams from three to four, scanned in a square pattern with angles offset from nadir at either $22.5^{\circ}$ or $7.5^{\circ}$. The intent of this change is to provide some redundancy in the measurements, and to allow for more measurements per time to get a better understanding of terrain features. The scanner can also change the number of beams and the beam angles on-the-fly, a capability the current NASA system does not have.

\subsection{Transceiver Simulation}

The lidar transceiver consists of four main parts. First, the laser transmitter is modelled, including parameters for up-chirp and down-chirp frequencies and durations, transmitted laser power, local oscillator power, and various losses in the transmitter optics and alignment errors. To allow for multiple targets in the beam footprint, for non-uniform beam profiles, and for beams impinging on a sloped surface, the beam footprint is sampled and each "beamlet" is modelled individually. ${ }^{5}$ The effect of this modelling for multiple returns or sloped surfaces is spread in the spectrum of $f_{b}$.

The laser linewidth effects are also modelled. The linewidth is assumed to be Gaussian in spectral shape. ${ }^{8}$ The effects of a non-zero linewidth are to spread out the Doppler and the up- and down-chirp frequency spectra. In particular, due to the relationship between radial speed, Doppler frequency, and wavelength,

$$
f_{d}=\frac{2 v_{r}}{\lambda},
$$

as $v_{r}$ increases, so does the Doppler spectral spread and the corresponding spread of the $f^{+}$and $f^{-}$spectra at the mixer output. 
The second part of the simulation includes the modelling of the lidar range equation, atmospheric backscatter and losses (appropriate for Mars), and solar background radiation. The atmospheric losses are not modelled with a detailed atmospheric model to maintain the goal of a fast simulation, however, a simple first-order model is used. During this step, the interaction of the sampled beam footprint with a sloped surface or multiple targets of different ranges (such as a bolder above the ground, or a cliff edge) is computed.

The third part of the transceiver simulation includes the receiver, consisting of the balanced diode detector and the resulting mixing process, the low-noise transimpedance amplifier, and any additional amplification. The current out of the detector from the mixing process is given by ${ }^{9}$

$$
I_{s i g} \propto \sqrt{2 P_{L O} P_{s i g}} \cos (M(t)) \cos \left(M\left(t-T_{d}\right)\right) \sin \left[\theta_{\text {sig }}(t)-\theta_{L O}(t)\right]
$$

where $\theta_{\text {sig }}(t)$ and $\theta_{L O}(t)$ are random phase errors, and

$$
M^{+}(t)=2 \pi\left[F_{1}+\frac{B}{2 T} t\right] t, \quad M^{-}(t)=2 \pi\left[F_{2}-\frac{B}{2 T} t\right] t
$$

for the up-chirp and down-chirp modulations, respectively. The resulting chirp frequency spectra that are not filtered by the receiver electronics become

$$
f^{+}= \begin{cases}\cos \left(2 \pi\left[\left(F_{1} T_{d}-\frac{B}{2 T} T_{d}^{2}-f_{d} T_{d}\right)+\left(f_{b}-f_{d}\right) t\right]\right) & f_{d}<f_{b} \\ \cos \left(2 \pi\left[-\left(F_{1} T_{d}-\frac{B}{2 T} T_{d}^{2}-f_{d} T_{d}\right)+\left(f_{d}-f_{b}\right) t\right]\right) & f_{d}>f_{b}\end{cases}
$$

and

$$
\left.f^{-}=\cos \left(2 \pi\left[-\left(F_{2} T_{d}+\frac{B}{2 T} T_{d}^{2}\right)+f_{d} T_{d}\right)+\left(f_{b}+f_{d}\right) t\right]\right)
$$

where $v_{r}>0$ (moving towards target), $f_{b}=\frac{B}{T} T_{d}$, and $T_{d}$ is the time delay of the return chirp. For the case $v_{r}<0$, the chirp frequency spectra are

$$
f^{+}=\cos \left(2 \pi\left[\left(F_{1} T_{d}-\frac{B}{2 T} T_{d}^{2}+f_{d} T_{d}\right)+\left(f_{b}+f_{d}\right) t\right]\right)
$$

and

$$
f^{-}=\left\{\begin{array}{ll}
\cos \left(2 \pi\left[-\left(F_{2} T_{d}+\frac{B}{2 T} T_{d}^{2}-f_{d} T_{d}\right)+\left(f_{b}-f_{d}\right) t\right]\right) & f_{d}<f_{b} \\
\cos \left(2 \pi\left[\left(F_{2} T_{d}+\frac{B}{2 T} T_{d}^{2}-f_{d} T_{d}\right)+\left(f_{d}-f_{b}\right) t\right]\right) & f_{d}>f_{b}
\end{array},\right.
$$

Unlike with FMCW radar systems, the very short wavelength of the lidar light $(\approx 1550 \mathrm{~nm})$, it is often the case that $f_{d}>f_{b}$, and a small change in velocity results in a large change in Doppler frequency. Note that the mixer output $f^{+}$and $f^{-}$may contain multiple frequencies due to multiple targets or slopes in the beam footprint. In the simulation, the actual time-domain cosine waveforms are not computed, but the frequency, phase, and magnitude of each cosine in the spectrum is. The mixed signals $f^{+}$and $f^{-}(5)-(8)$ above are then passed through the transimpedance amplifier model frequency response and any other amplifier model frequency responses included in the receiver electronics simulation. These simulated analog signals are then passed to the final part of the simulation.

The final step is to simulate the digital signal processing used to find the range and radial speed of the target(s) within the beam footprint. In the lidar hardware, the analog signal is digitized, and an FFT is used to detect the chirp frequencies. Since one of the goals of LadarSIM is to provide a fast, engineering-level simulation, it was necessary to simulate the spectral leakage, spreading, and scalloping loss effects of the FFT without actually generating the time-domain signals and computing the FFT. This is because the FFTs in the system are of length $2^{17}$, and computing one FFT for each segment of the modulation and for each measurement would require an extensive amount of computation for any reasonable simulation. The simulation of the FFT includes spectral leakage effects of a rectangular window, or a Hanning or Hamming window for sidelobe suppression, as selected by the user.

The simulation is performed by convolving the spectra of the mixed signals in the frequency domain with the frequency response of the FFT window function (or the appropriate Hanning or Hamming window function). 
This is computed using fast convolution with much smaller FFTs (typically $N=2048$ or $N=4096$ ) of the range of frequencies where the true spectra lie, and the FFT of the FFT window response. It is done with a much higher sampling rate in the frequency domain than the FFT bin spacing, and the result of the convolution is sampled at the appropriate frequencies for the FFT bin centers.

Once the FFT result is simulated, different detection methods can be investigated. Currently, a simple method is used, where the peak magnitude bin of the returned signal is found in each FFT (up-chirp, Doppler, and down-chirp). The sign of $v_{r}$ is found using the test that if $\left|f_{\text {max }}^{+}\right|>\left|f_{\text {max }}^{-}\right|$, the lidar is moving away from the target, otherwise the lidar is moving toward the target. From (5)-(8), the FFT chosen for detection is the one containing the spectra $f_{b}+f_{d}$, and the peak frequency of the Doppler FFT is subtracted from each of the frequency bins in that FFT. This process creates a set of frequency bins shifted by the estimated beat spectra $f_{b}$.

Finally, detection is performed by finding the peak magnitude frequency bin in each set of contiguous samples above $30 \%$ of the peak value. This threshold was chosen to eliminate candidate detections caused by FFT sidelobes. Since there might be more than one target in the beam footprint, each group of contiguous samples is considered. Once the peak magnitude(s) are found, the bin value(s) are tested for detection.

Detection is declared using a simulation of the statistics of the noise in the bin containing a signal. To compute these statistics, the shot noise generated by the signal, solar background, and atmospheric backscatter are combined with the noise generated by the detectors, transimpedance amplifier and other amplifiers as described by Neilsen, ${ }^{4}$ to give an estimate of the noise power resulting from the receiver electronics. This noise is used to estimate the Ricean statistics for the magnitude of the signal in the peak magnitude bin of the FFT, ${ }^{10}$ where the Ricean parameter is given by

$$
\sigma_{|X|}^{2}=\frac{E_{w} \sigma_{x}^{2}}{2}
$$

$x$ is the analog noise variance (including ADC noise), and $|X|$ is the FFT bin magnitude. The probability of detection is given by

$$
P_{d}=Q_{1}\left(\frac{r}{\sigma_{|X|}}, \frac{T h}{\sigma_{|X|}}\right)
$$

where $Q_{1}$ is the Marcum Q-function, $r$ is the bin magnitude, and $T h$ is the detection threshold, set by the user. Currently, LadarSIM uses the assumption that a detection must be found in all three segment FFTs in order to prevent a measurement dropout. Similarly, the probability of false alarm in a bin is given by Rayleigh statistics with the same parameter $\sigma_{|X|}^{2}$.

With the simplified detection method currently implemented, the range and speed error are determined by the fact that the range and velocity are both quantized to the nearest bin of the FFT. Using a uniform distribution of the bin quantization, the expected range error standard deviation is $\sigma_{R}=9.9 \mathrm{~cm}$ and the velocity error is $\sigma_{v}=1.024 \mathrm{~mm} / \mathrm{s}$ for the NASA lidar simulation.

One advantage of the design of LadarSIM is that the detection processing of the FFT outputs can be easily changed to allow for investigation of better methods to improve range and speed accuracy or improve the detection probability.

\section{EXAMPLE POINT CLOUDS}

\subsection{FMCW Doppler Transceiver Test}

To illustrate the behavior of the FMCW Doppler transceiver, a scenario to create a dense point cloud was run. The scenario includes a lidar moving forward with a level flight path at $250 \mathrm{~m}$ altitude, scanning in the cross-track direction (perpendicular to the level flight path) and looking down. The 3D scene is a small group of trees in the center of a hilly terrain. In this scenario the scanner creates two beams, one looking forward at an angle of 250 $\mu \mathrm{rad}$ and the other backward at an angle of $250 \mu \mathrm{rad}$. With this scenario, the forward beam should measure a slight positive radial velocity (toward lidar) and the backward beam should measure a slight negative velocity.

The results of the simulation are given in Figure 2. In Figure 2 (a), an example is given of the FFT bins simulated for a measurement with two objects in the beam footprint, without noise. The multiple detections 

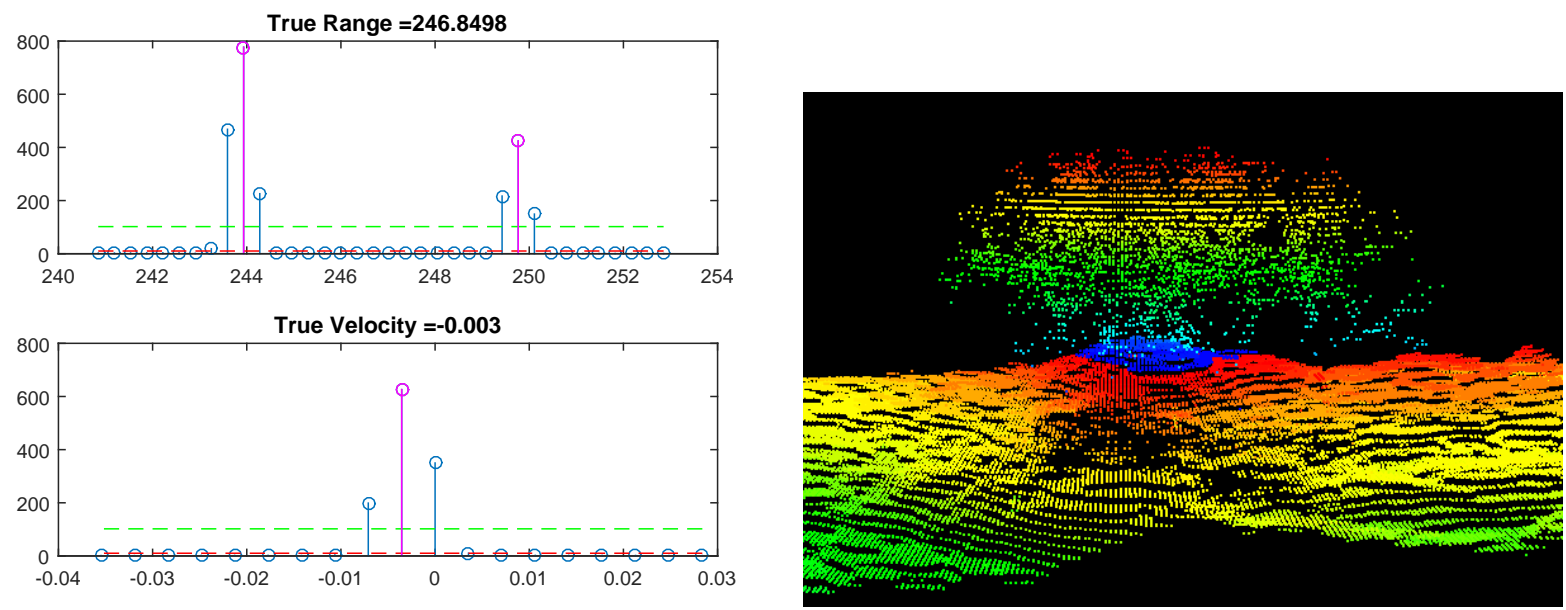

(a)

(b)

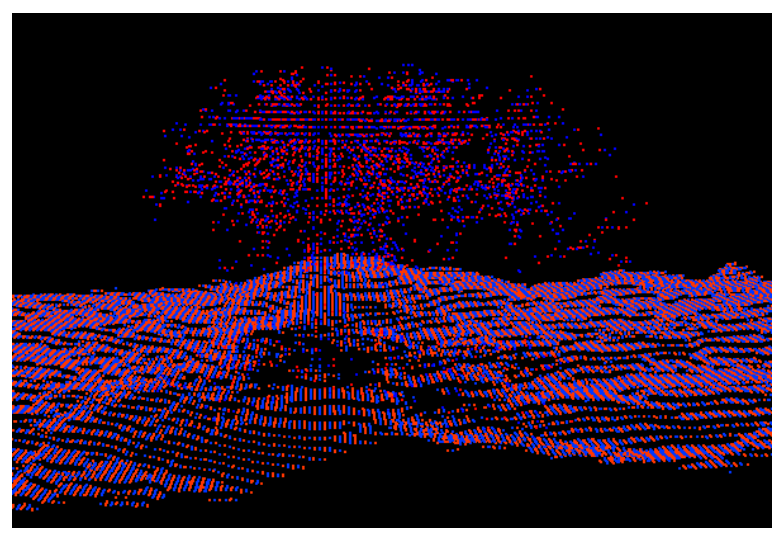

(c)

Figure 2: Simulation results for the dense point cloud scenario. (a) FFT bin values for measurement with two returns. The green dashed line represents the detection threshold, the red dashed line is the noise variance, and the maroon points are the detected measurements. A Hamming window was used. (b) Point cloud colored by range. (c) Point cloud colored by velocity.

occur because the lidar beam struck a tree in the scene and the ground below. The detection threshold is shown with a green dashed line, the Rayleigh noise variance is shown with a dashed red line, and the detections are colored in maroon. Note that the spectrum is spread to multiple bins due to a sub-optimal sampling rate for the chirp slope, and the effect of spectral spreading in the FFT.

The resulting point clouds are shown in Figures 2 (b) and 2 (c). In Figure 2 (b), the points have been colored by the measured range value, and in Figure 2 (c) the points are colored by measured velocity. As expected, the range bin quantization causes layers in the measurements. It is also interesting that the measurements from the beam pointed forward give small positive velocity values, and the beams pointing backward give small negative values, as indicated by the different colors.

\subsection{NASA Lidar Simulation Test}

The next simulation is intended to generate range and velocity data from a lidar descending to the terrain surface. To this end, the scenario consists of the terrain DEM scene used in Section 4.1 (with the trees removed), the 
four-beam scanner described in Section 3.2, and the lidar configuration described in Section 3.1. Examples of different trajectories were created, including straight down, and descending at a $45^{\circ}$ angle.

The results of the first simulation are given in Figure 3. In the scenario in Figure 3 (a), the lidar is pointing

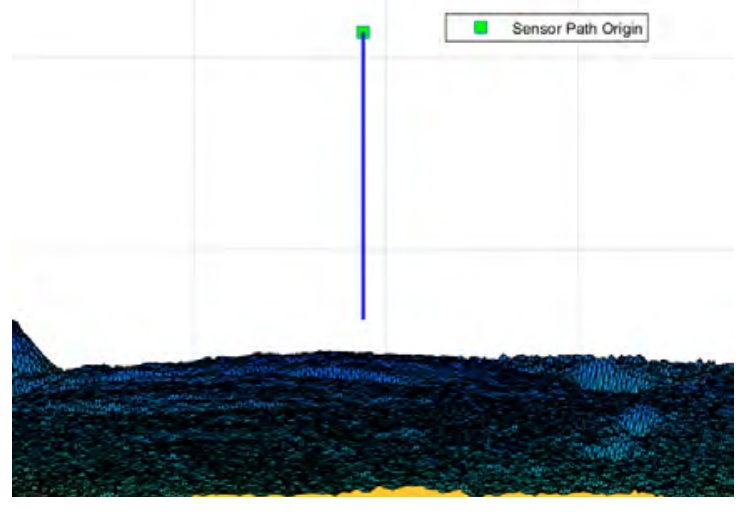

(a)

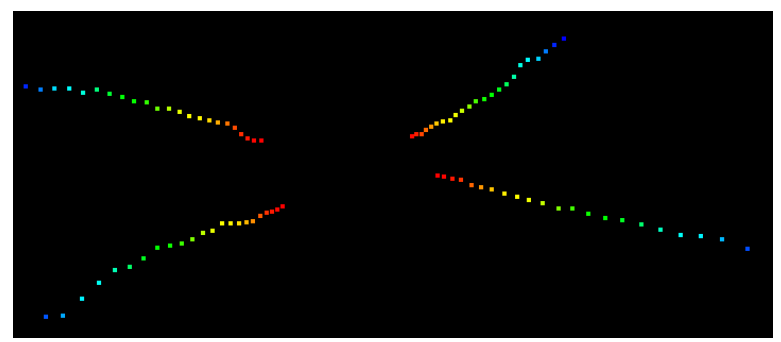

(b)

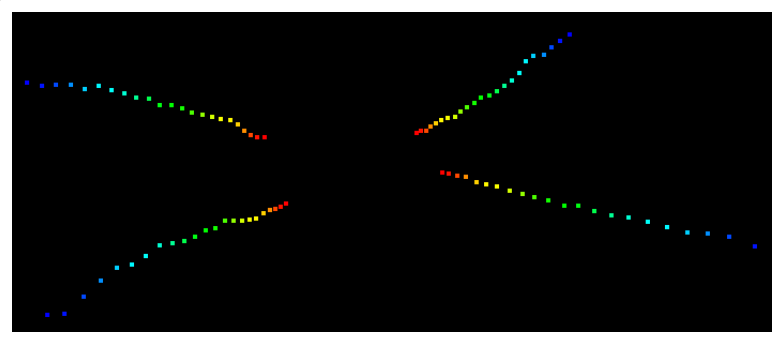

(c)

Figure 3: Simulation results for the high altitude descent. The scanner uses a four-beam configuration with each beam at a $22.5^{\circ}$ angle from nadir. (a) Path to terrain. (b) Point cloud colored by range. (c) Point cloud colored by velocity.

down and descending from $2000 \mathrm{~m}$ to $500 \mathrm{~m}$, starting with a speed of $100 \mathrm{~m} / \mathrm{s}$ and deceleration to $32 \mathrm{~m} / \mathrm{s}$. The measurements are made at a rate of one per second. The beams are offset by $22.5^{\circ}$ from nadir. As shown in the figure, the four points in each scan are located at the corners of a square on the terrain, with the square getting smaller as the lidar descends. Figure 3 (b) is colored by range, and shows a cycling through decreasing range measurements as the lidar descends. Figure 3 (c) is colored by velocity, and shows a cycling through decreasing velocity measurements as the lidar slows as it descends.

The simulation in Figure 4 is similar to Figure 3, but with a straight descent from $500 \mathrm{~m}$ to $100 \mathrm{~m}$, starting with a speed of $30 \mathrm{~m} / \mathrm{s}$ and decelerating to $6 \mathrm{~m} / \mathrm{s}$. These measurements are made at a rate of one per second. This regime of the descent will use the $7.5^{\circ}$ offset to improve the SNR for range measurements and to allow more points on the region directly below the lander to detect hazards.

The final simulation is a $45^{\circ}$ descent to the landing spot with the lidar looking down. The lidar is moving from $2000 \mathrm{~m}$ to $500 \mathrm{~m}$ in altitude, starting with a speed of $100 \mathrm{~m} / \mathrm{s}$, a deceleration to $30 \mathrm{~m} / \mathrm{s}$, and measurements at a rate of one per second. The point clouds are shown in Figure 5.

In this scenario, the lidar is moving with a lateral velocity, causing two of the beams to measure lower velocities, while two measure higher velocities. Note that the beams at the corners of a square are overlapping and growing smaller as the lander descends. 


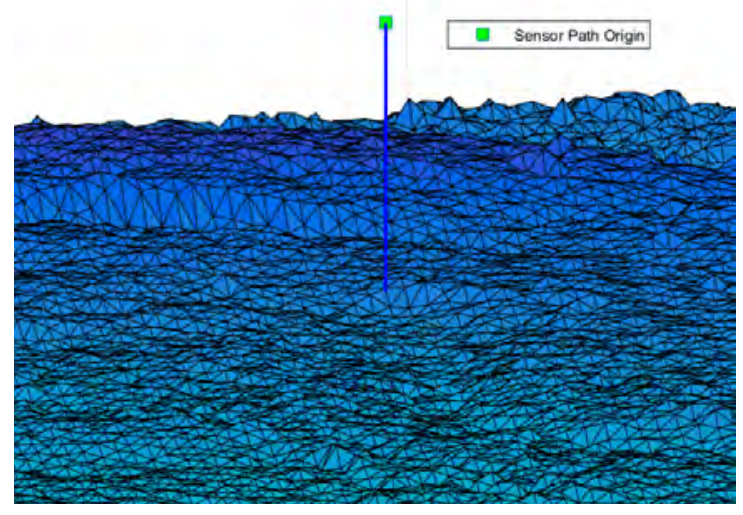

(a)

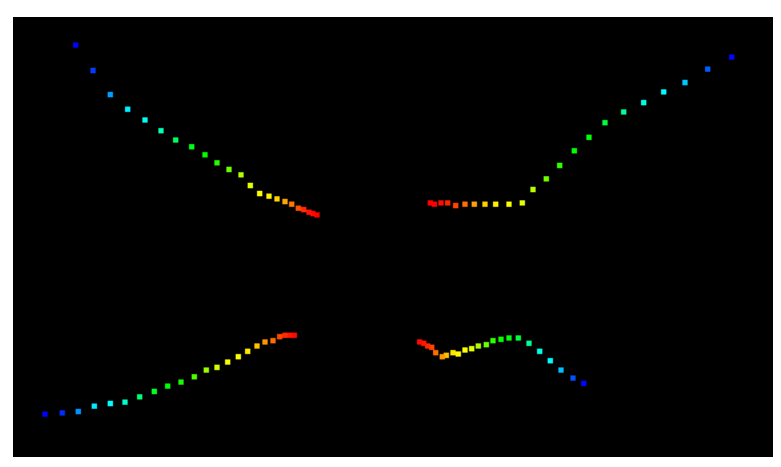

(b)

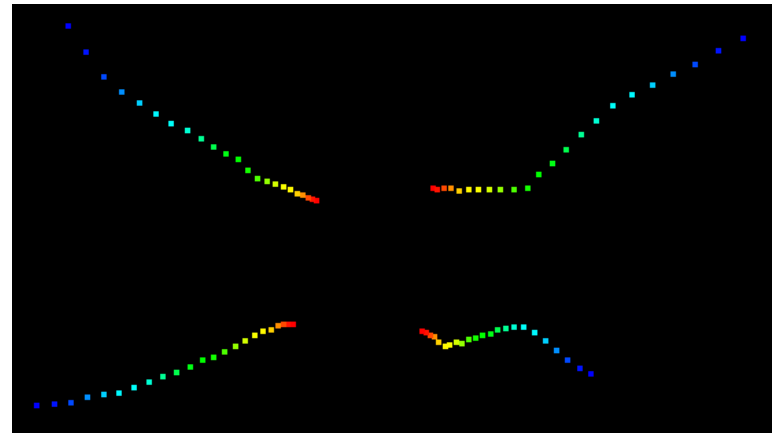

(c)

Figure 4: Simulation results for the low altitude descent. The scanner uses a four-beam configuration with each beam at a $7.5^{\circ}$ angle from nadir. (a) Path to terrain. (b) Point cloud colored by range. (c) Point cloud colored by velocity.

\section{CONCLUSIONS AND DISCUSSION}

The modification to LadarSIM to support FMCW Doppler lidar has opened up the ability to simulate lidar systems used for attitude determination, navigation, and control of planetary landers. From this simulated data, design parameters for the lidar hardware can be investigated, signal processing algorithms can be improved, and algorithms for determining altitude above terrain and lander velocity can be developed. In addition, the optimal configuration for different numbers and orientations of multiple beams can be investigated, both for velocity determination and to investigate the ability to detect hazards below the lander. LadarSIM provides a very flexible, engineering design-level simulation of these tradeoffs.

There are aspects of simulation that need to be added to the LadarSIM FMCW Doppler simulation. For example, the effect of false alarms is not yet implemented, since these are very infrequent for the current NASA lidar design. As detection thresholds are optimized to prevent dropouts in low SNR situations, false alarms become a larger problem.

In addition, the effects of phase distortions such as speckle need to be added to LadarSIM. Empirical evidence points to the hypothesis that speckle-induced dropouts are much more common that dropouts due to low SNR in the lidar returns.

Finally, the simple detection method currently implemented in LadarSIM can be improved using signal processing techniques which combine the measurement in both the up-chirp and down-chirp signals. 


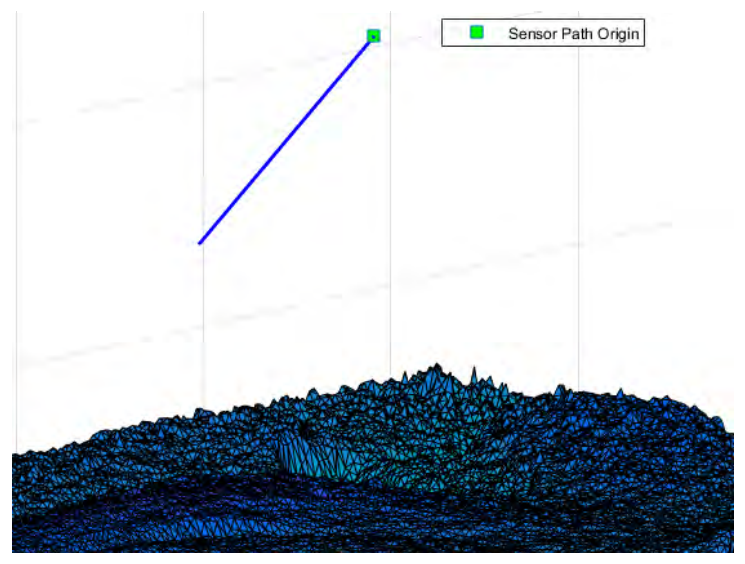

(a)

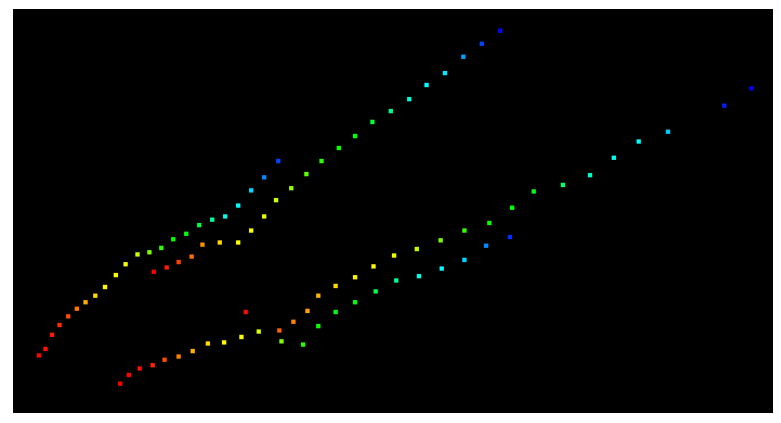

(b)

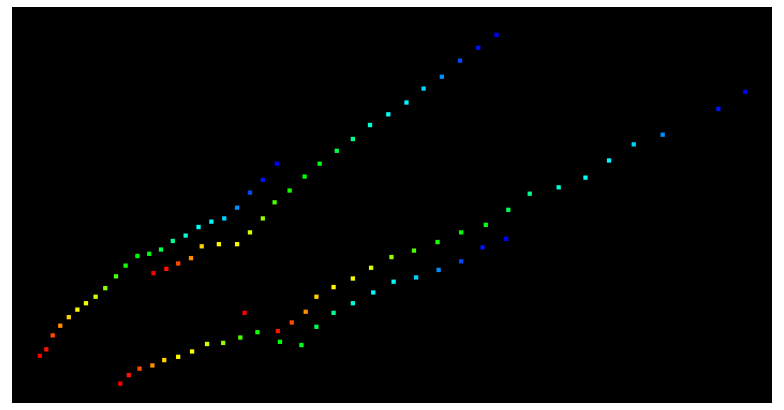

(c)

Figure 5: Simulation results for the $45^{\circ}$ high altitude descent. The scanner uses a four-beam configuration with each beam at a $22.5^{\circ}$ angle. (a) Path to terrain. (b) Point cloud colored by range. (c) Point cloud colored by velocity.

\section{REFERENCES}

[1] Amzajerdian, F., Pierrottet, D. F., Hines, G. D., Petway, L. B., and Barnes, B. W., "Doppler lidar sensor for precision navigation in GPS-deprived environment," in [Laser Radar Technology and Applications XVIII], 8731, 8731G-1-8731G-6, SPIE (2013).

[2] Amzajerdian, F., Petway, L., Hines, G., Barnes, B., Pierrottet, D., and Lockard, G., "Doppler lidar sensor for precision landing on the moon and mars," in [Aerospace Conference, 2012 IEEE], 1-7 (Mar. 2012).

[3] Pierrottet, D., Amzajerdian, F., Petway, L., Barnes, B., Lockard, G., and Hines, G., "Navigation doppler lidar sensor for precision altitude and vector velocity measurements: flight test results," Proc. SPIE 8044, 80440S-80440S-11 (2011).

[4] Neilsen, K. D., Budge, S. E., Pack, R. T., Fullmer, R. R., and Cook, T. D., "Design and validation of the eyesafe LADAR test-bed (ELT) using the LadarSIM system simulator," in [Laser Radar Technology and Applications XIV], Turner, M. D. and Kamerman, G. W., eds., 7323, 73230B, SPIE, Orlando, FL, USA (May 2009).

[5] Budge, S. E., Leishman, B. C., and Pack, R. T., "Simulation and modeling of return waveforms from a ladar beam footprint in USU LadarSIM," in [Laser Radar Technology and Applications XI], Kamerman, G. W. and Turner, M. D., eds., 6214, 62140N, SPIE, Orlando (Kissimmee), FL, USA (Apr. 2006).

[6] Brooker, G. and Brooker, A. T., [Introduction to sensors for ranging and imaging], SciTech Pub. Incorporated (2009). 
[7] Adany, P., Allen, C., and Hui, R., "Chirped lidar using simplified homodyne detection," J. Lightw. Technol. 27, 3351-3357 (Aug. 2009).

[8] Domenico, G. D., Schilt, S., and Thomann, P., "Simple approach to the relation between laser frequency noise and laser line shape," Appl. Opt. 49, 4801-4807 (Sept. 2010).

[9] Adany, P., Simplified Homodyne Detection for FM Chirped LIDAR, Master's thesis, University of Kansas (Nov. 2007).

[10] Richards, M. A., "The discrete-time Fourier transform and discrete Fourier transform of windowed stationary white noise," Georgia Institute of Technology, Tech. Rep (2013). 